\title{
Electronic properties of bilayer phosphorene quantum dots in the presence of perpendicular electric and magnetic fields
}

\author{
L. L. Li, ${ }^{1,2,{ }^{*}}$ D. Moldovan,,${ }^{1, \dagger}$ W. Xu ${ }^{2,3}$ and F. M. Peeters ${ }^{1, \dagger}$ \\ ${ }^{1}$ Department of Physics, University of Antwerp, Groenenborgerlaan 171, B-2020 Antwerpen, Belgium \\ ${ }^{2}$ Key Laboratory of Materials Physics, Institute of Solid State Physics, Chinese Academy of Sciences, Hefei 230031, China \\ ${ }^{3}$ Department of Physics, Yunnan University, Kunming 650091, China
}

(Received 1 August 2017; revised manuscript received 13 September 2017; published 10 October 2017)

\begin{abstract}
Using the tight-binding approach, we investigate the electronic properties of bilayer phosphorene (BLP) quantum dots (QDs) in the presence of perpendicular electric and magnetic fields. Since BLP consists of two coupled phosphorene layers, it is of interest to examine the layer-dependent electronic properties of BLP QDs, such as the electronic distributions over the two layers and the so-produced layer-polarization features, and to see how these properties are affected by the magnetic field and the bias potential. We find that in the absence of a bias potential only edge states are layer polarized while the bulk states are not, and the layer-polarization degree (LPD) of the unbiased edge states increases with increasing magnetic field. However, in the presence of a bias potential both the edge and bulk states are layer polarized, and the LPD of the bulk (edge) states depends strongly (weakly) on the interplay of the bias potential and the interlayer coupling. At high magnetic fields, applying a bias potential renders the bulk electrons in a BLP QD to be mainly distributed over the top or bottom layer, resulting in layer-polarized bulk Landau levels (LLs). In the presence of a large bias potential that can drive a semiconductor-to-semimetal transition in BLP, these bulk LLs exhibit different magnetic-field dependences, i.e., the zeroth LLs exhibit a linearlike dependence on the magnetic field while the other LLs exhibit a square-root-like dependence.
\end{abstract}

DOI: 10.1103/PhysRevB.96.155425

\section{INTRODUCTION}

Two-dimensional (2D) black phosphorus (BP) is a directband-gap semiconducting material, which has been recently fabricated through exfoliation methods [1-3]. Bulk BP is a layered material in which individual single layers are stacked and coupled via the van der Waals interaction [4]. A single layer of BP is called phosphorene, where each atom is covalently bonded with three neighboring atoms via $s p^{3}$ hybridization, thereby forming a puckered hexagonal lattice [5]. Due to this unique lattice structure of phosphorene, 2D BP exhibits strongly anisotropic electronic, optical, and transport properties [6-8], which are atypical for most 2D materials. Encapsulation of 2D BP with hexagonal boron nitride leads to the formation of a 2D electron gas with high electron mobility, which allows the observation of magnetic quantum oscillations [9] and the quantum Hall effect [10]. One of the most striking characteristics of $2 \mathrm{D} \mathrm{BP}$ is its strong response to external strain and bias. It was shown that the electronic properties of monolayer and bilayer BP can be tuned by applying external strain and/or bias [11,12]. In particular, an external bias can drive a semiconductor-to-semimetal transition in bilayer BP [13,14], leading to the appearance of Dirac-like cones and paraboliclike bands (inverted) in its energy spectrum [15]. In addition to strain and bias, edge effects also play an important role in affecting the physical properties of 2D BP nanostructures. For instance, armchair- and zigzagterminated nanoribbons of 2D BP exhibit different scaling rules for the band gap versus the ribbon width $\left(E_{g} \sim 1 / W\right.$ and

\footnotetext{
*longlong.li@uantwerpen.be

†dean.moldovan@uantwerpen.be

francois.peeters@uantwerpen.be
}

$1 / W^{2}$, respectively, with $E_{g}$ the band gap and $W$ the ribbon width) [16].

Recently, BP quantum dots (QDs) have been fabricated through chemical methods $[17,18]$. The obtained QDs have a lateral size of several nanometers and a vertical thickness of few layers. Therefore, one may expect significant confinement and edge effects in such small nanoscale QDs. Motivated by these experiments, theoretical studies have been carried out on the electronic and optical properties of monolayer phosphorene (MLP) QDs [19-21]. Interesting results were obtained, such as unconventional midgap edge states [19], anomalous size dependence of optical emission gap [20], and robust magneto-optical absorption by edge states [21]. In addition to MLP QDs, the electronic properties of MLP quantum rings $(\mathrm{QRs})$ were also investigated recently and Aharonov-Bohm oscillations were predicted in the energy spectrum of such QRs [22]. Because the electronic, optical, and transport properties of 2D BP are also strongly dependent on its layer number [8,23-25], it is of both fundamental and practical interest to investigate the effect of the interlayer coupling on these physical properties. Bilayer phosphorene (BLP) is a natural candidate that can provide basic information on such an interlayer coupling effect.

In the present work, we investigate theoretically the electronic properties of BLP QDs in the presence of perpendicular electric and magnetic fields. Within the tight-binding (TB) approach, the energy levels, wave functions, density of states, and layer-dependent electronic properties of BLP QDs are obtained numerically as a function of perpendicular magnetic field and of perpendicular bias potential. The effects of the size of the QD (i.e., the confinement effect) and the type of edges on the electronic properties of BLP QDs are also investigated. Here, for simplicity, we consider square-shaped BLP QDs 
with zigzag and armchair edges as our model QDs. Although realistic BLP QDs could have more complex (irregular) shapes and edges, such simple model QDs may provide basic insights into important confinement and edge effects.

Magnetic-field effects such as Landau levels and magnetooptical and magneto-transport properties have been investigated in graphene QDs (GQDs) [26] as well as in bulk MLP and its nanoribbons [27]. However, the subject studied in the present work is different from those two works: (i) Compared to GQDs (Ref. [26]), BLP QDs and MLP QDs exhibit midgap edge states due to the finite band gap of BLP and MLP, the energy levels of which are quasiflat with increasing magnetic field, and they also exhibit anisotropic Landau levels formed by conduction/valence bulk states at high magnetic fields due to the anisotropic band structure of BLP and MLP. (ii) When compared to MLP (Ref. [27]), BLP shows some different features in the electronic energy spectrum due to the interlayer coupling effect and its QDs exhibit interesting layer-polarization features for edge and bulk states that can be influenced by perpendicular electric and magnetic fields. (iii) The effect of an external perpendicular electric field (i.e., the bias effect), not considered in Refs. [26,27], was included in the present paper.

The main results obtained in this work are as follows: (i) Distinctive edge and bulk states are present in BLP QDs and they exhibit different responses to perpendicular electric and magnetic fields. (ii) In small-size BLP QDs edge states may couple with bulk states. This bulk-edge coupling is not present in large-size BLP QDs and it decreases and eventually disappears with increasing magnetic field. (iii) Edge and bulk states in BLP QDs exhibit different layer-dependent electronic properties, such as layer-resolved electronic distributions and so-produced layer-polarization features, and these layerdependent properties can be manipulated by perpendicular electric and magnetic fields. In addition, magneto-electronic properties of unbiased and biased BLP QDs are analyzed in detail, which is essential for the understanding of other important physical properties, such as electrically tunable magneto-optical and magneto-transport properties.

This paper is organized as follows. In Sec. II, we present the TB model approach for studying the electronic properties of BLP QDs in the presence of perpendicular electric and magnetic fields. In Sec. III, we briefly investigate the effect of a bias potential on the electronic band structure of bulk BLP. In Sec. IV, the main results are presented and analyzed for the magnetic-field and bias-potential dependencies of layer-dependent electronic properties (i.e., energy levels, wave functions, and density of states) of BLP QDs. Finally, we make a summary and give concluding remarks in Sec. V.

\section{THEORETICAL APPROACH}

We consider AB-stacked (Bernal) BLP consisting of two phosphorene layers coupled via the van der Waals interaction, as shown in Fig. 1(a). This stacking configuration is energetically most stable for BLP according to first-principle calculations [28] and can be viewed as shifting the upper and lower phosphorene layers by half-unit-cell length along the armchair or zigzag direction. Due to the puckered lattice structure, phosphorene has two atomic sublayers and thus
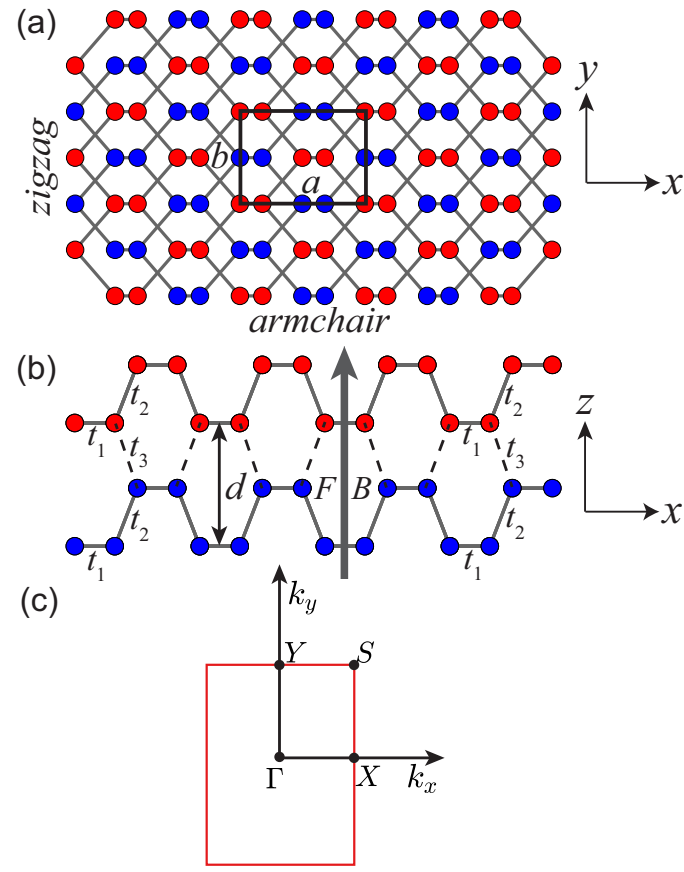

FIG. 1. (a) Top and (b) side views of the lattice structure of AB-stacked BLP. The upper and lower phosphorene layers, with interlayer separation $d$, are depicted by red and blue phosphorus atoms, respectively. The armchair (zigzag) edges are assumed to be along the $x(y)$ direction. External electric and magnetic fields, denoted by $F$ and $B$, are applied perpendicular to the phosphorene layers (i.e., along the $z$ direction). The black rectangle represents the unit cell of BLP with $a$ and $b$ being the lattice constants. The symbols $t_{1}$ and $t_{2}$ are the intralayer hopping parameters and $t_{3}$ is the interlayer hopping parameter. (c) Schematic plot of the first Brillouin zone (the red rectangle) with four high-symmetry points $\Gamma, X, Y$, and $S$ as indicated by black dots.

BLP has four atomic sublayers [see Fig. 1(b)]. Low-energy electrons and holes in BLP are described by the following TB Hamiltonian:

$$
H=\sum_{i} \varepsilon_{i} c_{i}^{\dagger} c_{i}+\sum_{i \neq j} t_{i j}^{\|} c_{i}^{\dagger} c_{j}+\sum_{i \neq j} t_{i j}^{\perp} c_{i}^{\dagger} c_{j},
$$

where the summation runs over all lattice sites of the system, $\varepsilon_{i}$ is the on-site energy at site $i, t_{i j}^{\|}\left(t_{i j}^{\perp}\right)$ is the intralayer (interlayer) hopping energy between sites $i$ and $j$, and $c_{i}^{\dagger}$ $\left(c_{j}\right)$ is the creation (annihilation) operator of an electron at site $i(j)$. This TB model has been proposed for MLP and BLP [29-31], and has been shown to accurately reproduce the band structures of MLP and BLP obtained from DFT- $G W$ calculations over a wide energy range. However, as pointed out by the previous works $[29,32,33]$, the main features of the band structure of MLP can be qualitatively described by a minimal TB model that only takes into account the two largest hopping parameters $\left(t_{1}\right.$ and $\left.t_{2}\right)$. For BLP, additional hopping parameters are required to describe the interlayer coupling effect, and in a minimal TB model only the nearestneighbor interlayer hopping parameter $\left(t_{3}\right)$ needs to be taken into account since it crucially determines the band gap of BLP. With these hopping parameters $\left(t_{1}, t_{2}\right.$, and $\left.t_{3}\right)$, the band gap can be evaluated as $E_{g}=2 t_{2}+4 t_{1}$ for MLP and 
$E_{g}=2 t_{2} \sqrt{1+\left(t_{3} / t_{2}\right)^{2}}+4 t_{1}-2 t_{3}$ for BLP. From these two expressions, it can be seen that the band gap of BLP is smaller than that of MLP, which is induced by the third hopping parameter $t_{3}$ characterizing the interlayer coupling effect in BLP. We found that by choosing $t_{1}=-1.21 \mathrm{eV}, t_{2}=3.18 \mathrm{eV}$, and $t_{3}=0.22 \mathrm{eV}$ the band gaps of MLP and BLP calculated from this minimal TB model are given by $E_{g}^{\mathrm{MLP}}=1.51 \mathrm{eV}$ and $E_{g}^{\mathrm{BLP}}=1.09 \mathrm{eV}$, which agree with those obtained by the full TB model that includes five intralayer hoppings for MLP and additional four interlayer hoppings for BLP [29]. Moreover, the corresponding band structures of MLP and BLP near their band gaps are also found to be in agreement with those obtained by the full TB model. These motivate us to employ this three-parameter TB model to study the low-energy electronic properties of BLP.

In the presence of a perpendicular electric field, the two atomic sublayers in MLP gain different electrostatic on-site potential energies in the form of $-V / 2$ and $V / 2$, while the four atomic sublayers in BLP gain different electrostatic on-site potential energies in the form of $(1 / 2+\xi) V,(1 / 2-\xi) V$, $(-1 / 2+\xi) V$, and $(-1 / 2-\xi) V$, where $V=e F d$ is the bias potential energy across the top and bottom phosphorene layers, with $e$ the elementary charge, $F$ the electric-field strength, $d$ the interlayer separation, and $\xi=0.202$ a linear scaling factor that accounts for the sublayer dependence of the on-site electrostatic potential [14]. The effect of applying a perpendicular magnetic field to BLP is incorporated into the TB Hamiltonian (1) via the Peierls substitution, which modifies the intralayer and interlayer hopping energies as $t_{i j}^{\|, \perp} \rightarrow t_{i j}^{\|, \perp} \exp \left[i\left(2 \pi / \Phi_{0}\right) \int_{i}^{j} \mathbf{A} \cdot d \mathbf{l}\right]$, where $\Phi_{0}=h / e$ is the magnetic flux quantum with $h$ the Planck constant, and $\mathbf{A}=(0, B x, 0)$ is the magnetic vector potential in the Landau gauge with $B$ the magnetic-field strength. The magnetic flux threading a plaquette is defined as $\Phi=B a b$ in units of the flux quantum $\Phi_{0}$, with $a=4.37 \AA$ and $b=3.31 \AA$ the two in-plane lattice constants of phosphorene.

In principle, one has to consider for bulk BLP the charge distribution over the top and bottom phosphorene layers under an external perpendicular electric field. The charge distribution produces an internal electric field that counteracts the external one, which in turn influences the electronic band structure of BLP and so the charge distribution over the two phosphorene layers. This charge redistribution may affect the interlayer coupling strength because it may change the interlayer electronic hopping distance. In order to account for such a charge redistribution effect, a self-consistent calculation based on a band-structure model (e.g., DFT or TB) and the Poisson equation is needed. The charge screening depends on its densities in the two layers, which can reduce the external electric field applied and can be obtained from the self-consistent calculation. A rough estimate of the chargescreening-induced potential energy gives a value of the order of tens of meV for layer charge density of $10^{12} \mathrm{~cm}^{-2}$, which is less than the external bias potential energy of $1 \mathrm{eV}$. Furthermore, as we consider QDs in BLP, the charge screening effect should be even smaller in such nanostructures because the layer charge densities are much smaller as compared to those in bulk (infinite) BLP. Previous theoretical works on bulk BLP $[30,31]$ that did not consider the charge screening effect in the
TB model give good agreement with DFT calculations. There are also other screening effects, such as substrate screening, which may be important but are not relevant to the present work where we considered QDs in pristine BLP. Therefore, the present TB model is qualitatively valid to describe the effect of an external perpendicular electric field on the electronic properties of BLP QDs.

BLP QDs can be modeled by cutting an infinite BLP sheet into small-area flakes with various geometric shapes (e.g., rectangle, triangle, hexagon, and circle) and with different edge types (e.g., zigzag, armchair, and disordered). Here, for simplicity, we consider square-shaped BLP QDs with zigzag and armchair edges as our model QDs. Although realistic BLP QDs could have more complex (irregular) shapes and edges, such simple model QDs may provide basic insights into crucial edge and confinement effects. The energy levels and wave functions of square-shaped BLP QDs subjected to perpendicular electric and magnetic fields are obtained by numerically solving the TB model. All numerical TB calculations are performed using the recently developed PYBINDING package [34].

With the energy levels obtained, the electronic density of states is computed as $\operatorname{DOS}(E)=\sum_{n} \exp \left[-\left(E-E_{n}\right)^{2} / \Delta^{2}\right]$, with $E$ the given energy, $n$ the state index, $E_{n}$ the energy level, and $\Gamma$ the broadening factor. In the present work, unless otherwise specified, $\Delta=5 \mathrm{meV}$ is adopted throughout the DOS calculations.

\section{BULK BLP: BIAS EFFECT}

Before diving into BLP QDs, we first consider the effect of a perpendicular electric field (i.e., the bias effect) on the band structure of bulk BLP. Due to the in-plane translational invariance, a Fourier transform is performed to convert the real-space TB Hamiltonian (1) into momentum space, and then the corresponding Hamiltonian is numerically diagonalized to obtain the band structure.

In Figs. 2(a)-2(d), we show the band structure of bulk BLP for different bias potential energies $V$ as indicated in (a)-(c) and the band energies at the $\Gamma$ point as a function of $V$ in (d). As can be seen, unbiased BLP has an anisotropic band structure with a finite direct band gap, which is inherently due to the puckered lattice structure of phosphorene. Applying a bias reduces the band gap of BLP and eventually drives a semiconductor-to-semimetal transition. The critical bias potential energy for such a transition is found to be $V_{c} \simeq$ $1.5 \mathrm{eV}$, in agreement with recent theoretical work [13]. In the semimetal phase, biased BLP exhibits an interesting anisotropic band structure: it is linearlike and gapless in the $\Gamma-Y$ direction, but quadraticlike and inverted in the $\Gamma-X$ direction. Consequently, Dirac-like cones, paraboliclike bands, and band inversions coexist in biased semimetallic BLP. For comparative purposes, we also show in Figs. 2(e)-2(h) the effect of a perpendicular electric field on the band structure and the corresponding $\Gamma$-point band energies of bulk MLP: (e)-(g) for the band structure and (h) for the $\Gamma$-point band energies. As can be seen, applying a bias potential only increases the band gap of MLP while it has little effect on the main characteristics of the band dispersion, in sharp contrast to the case of BLP. This 



FIG. 2. Band structures of infinite BLP (MLP) subjected to different bias potential energies $V$ as indicated in (a)-(c) [(e)-(g)] and the band energies at the $\Gamma$ point as a function of $V$ shown in (d) [(h)]. The red dashed line denotes the zero-energy reference.

large difference is attributed to the interlayer coupling effect in BLP, which is also responsible for the band-gap decrease in BLP as compared to that in MLP. The electrically tunable band structure of BLP is expected to have important consequences on the electronic properties of biased BLP QDs in the presence of a perpendicular magnetic field.

It should be noted that in Fig. 2 the bias potential energy $V$ is set in the range of $0-2 \mathrm{eV}$. For a typical value of $V=1 \mathrm{eV}$, a rough estimate using the relation $V=e F d(d=0.523 \mathrm{~nm}$ is the interlayer separation of BLP) gives a corresponding electric field $F$ as high as $20 \mathrm{MV} / \mathrm{cm}$. It is known that generating such a huge electric field is a big challenge for experiments, which could possibly be generated by ionic gating and/or polarized interfaces as proposed in Ref. [35]. On the other hand, there are a large number of theoretical studies on phosphorene based on TB or DFT calculations that consider an external electric field of this magnitude or even larger [13,14,30,31]. Experimentally, strong electric fields are also commonly used to study the electrical response of material properties. For instance, the vibrational Stark effects were measured by applying an external electric field of order $1 \mathrm{MV} / \mathrm{cm}$ to an organic compound containing the vibrational probe of interest [36]. Moreover, it was already demonstrated that the magnitude of the gate-induced electric field can reach up to the order of 1 $\mathrm{MV} / \mathrm{cm}$ in micrometer-scale samples [37], which is expected to be even larger in nanostructures due to the reduced screening.

\section{BLP QD: MAGNETIC AND BIAS EFFECTS}

Now we turn to the study of the electronic properties of BLP QDs in the presence of perpendicular magnetic field and bias potential. Because the finite size breaks in-plane translational invariance, the real-space TB Hamiltonian (1) is directly diagonalized to obtain the eigenenergies and eigenfunctions of the electronic states in BLP QDs. In the following, the effects of magnetic field and bias potential on the electronic properties of such QDs are investigated.

\section{A. Magnetic-field effect}

In Figs. 3(a) and 3(b), we show the DOS-projected energy levels of an unbiased BLP QD (i.e., $V=0$ ), as a function of the magnetic flux $\Phi$, for different dot sizes $L$ : (a) $L=3.5 \mathrm{~nm}$ and (b) $L=7.5 \mathrm{~nm}$. Because of the small size of the QD, a large magnetic field ( $B=2850$ T for $\Phi=0.1 \Phi_{0}$ ) is required in order to produce a significant influence on the energy levels. Nevertheless, as the influence of the magnetic field scales with the magnetic flux threading the QD, similar results will be obtained for smaller magnetic fields if larger-size QDs are considered. Such large magnetic fields have also been considered in the TB modeling of phosphorene confined systems [19,38].

As can be seen in Figs. 3(a) and 3(b), there are nearly flat energy levels within the band gap of the BLP QD. These energy levels correspond to the edge states, while those above (below) the band gap correspond to the conduction (valence) bulk states. Here we distinguish edge and bulk states in terms of their wave-function properties: the former are strongly localized at the QD boundary while the latter are mainly distributed around the QD center [see Figs. 3(c)-3(e)], which show the probability densities $|\Psi|^{2}$ of the electronic states indicated by points 1-3 in Fig. 3(a). An important feature of the edge states is that they are almost unaffected by the magnetic field, as reflected by the quasiflat energy levels shown in Figs. 3(a) and 3(b). This feature is attributed to the strong localized nature of the edge states. Furthermore, we find that the DOS of the edge states is larger than that of the bulk states. This is because the edge levels are spaced very closely to each other and thus they can be viewed as nearly degenerate.

However, when comparing Figs. 3(a) and 3(b), the following differences can be observed: (i) The smaller-size QD has a larger band gap and also a larger energy-level separation due to the stronger confinement effect. (ii) The larger-size QD has a larger electronic DOS for both the bulk and edge states. This is because the bulk (edge) DOS is proportional to the number of atoms in the QD center (at the zigzag boundary) and thus both of them increase with the dot size. (iii) At high 

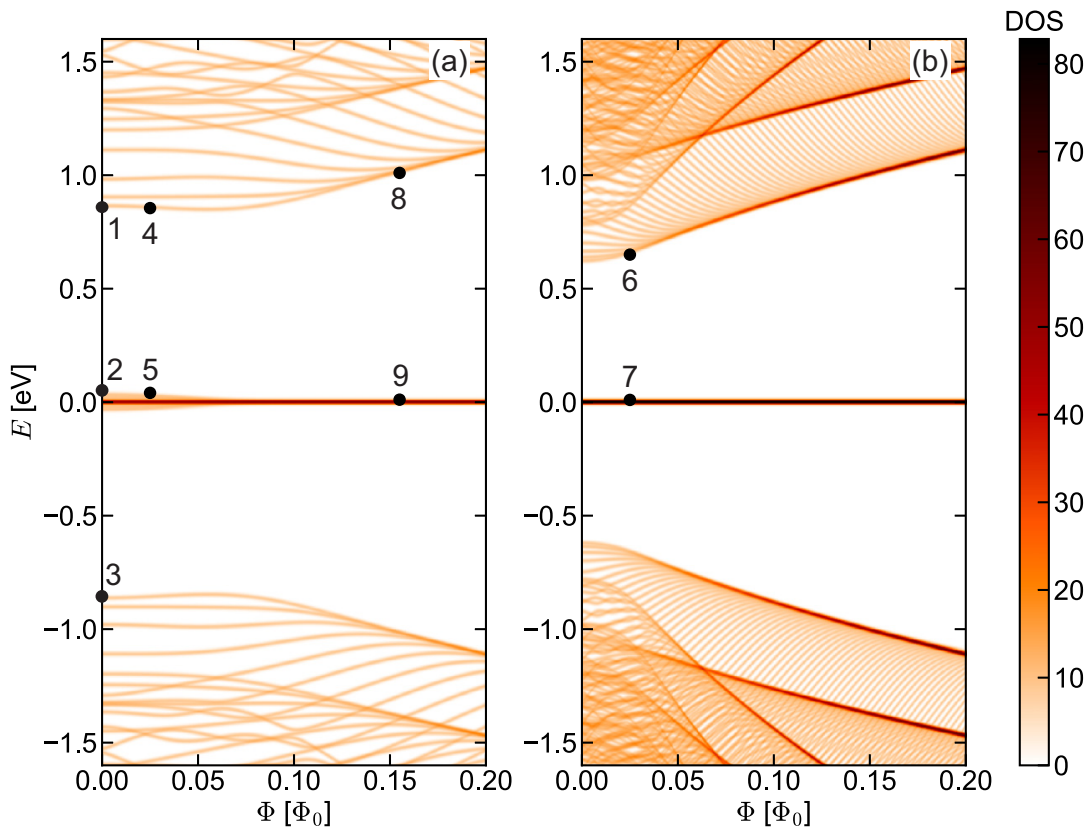
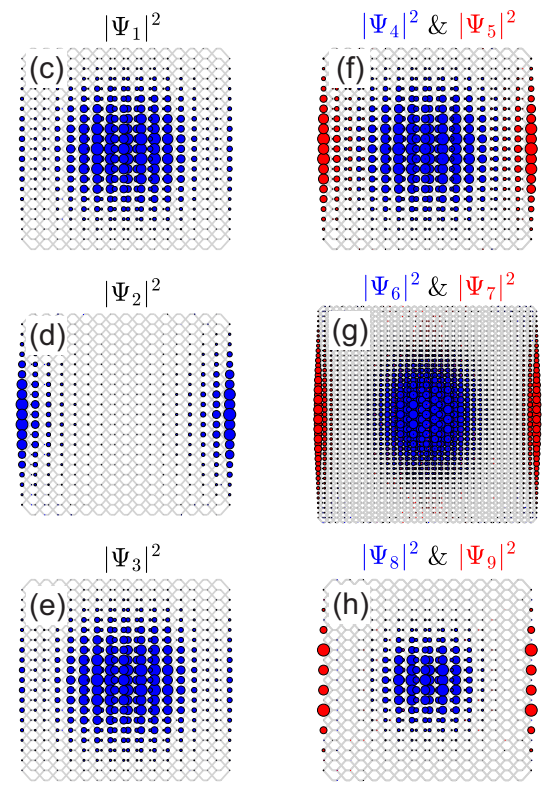

FIG. 3. (a) [(b)] Energy levels of an unbiased BLP QD, with dot size $L=3.5(7.5) \mathrm{nm}$, as a function of the magnetic flux $\Phi$, where the colorbar shows the DOS values at these levels. (c)-(e) Probability densities of the electronic states denoted by points 1-3 shown in (a). (f)-(h) Probability densities of the electronic states denoted by points 4-9 shown in (a) and (b). The size of the blue and red dots shown in (c)-(h) represents the amplitude of the electronic probability density.

magnetic fields, the bulk levels in the larger-size QD converge to distinct Landau levels (LLs) with a large DOS due to the high LL degeneracy. (iv) The edge states in the smaller-size QD are less unaffected by the magnetic field and have a certain bandlike broadening at lower magnetic fields. This broadening comes from the bulk-edge coupling in the smaller-size QD, while it is absent in the larger-size QD where the edge states have no such bandlike broadening. Our numerical calculations indicate that similar bulk-edge coupling can also be observed in small-size MLP QDs.

To see clearly the presence (absence) of the bulk-edge coupling in the smaller-sized (larger-sized) QD, we show in Figs. 3(f) and 3(g) the probability densities of the bulk and edge states denoted by points 4 and 5 in Fig. 3(a) and those denoted by points 6 and 7 in Fig. 3(b). We also find that the bulk-edge coupling in the smaller-size QD becomes much weaker at high magnetic fields [see Fig. 3(h)], which shows the probability densities of the bulk and edge states denoted by points 8 and 9 in Fig. 3(a). This is because the bulk states are more confined around the QD center at high magnetic fields due to the strong magnetic confinement.

We have to point out that the edge states predicted here will vanish in phosphorene nanoribbons and QDs with edge bond termination (passivation), as demonstrated by DFT calculations [39]. Therefore, such edge-state induced phenomena are likely to be invisible in the experiments. However, from a theoretical point of view, such edge states can still survive as long as there are zigzag boundaries assumed in the model system. Furthermore, such well-defined boundaries may possibly be realized in the future because of continuously improved nanofabrication techniques, as was already demonstrated for the case of graphene nanoribbons [40].
Since BLP is made of two coupled phosphorene layers with each one having two atomic sublayers, it is natural to think of studying the electronic-state distributions over these two atomic layers or four atomic sublayers. In doing so, we need to look into the layer-resolved electronic probability densities for both the bulk and edge states. Therefore, we define a new physical quantity $\eta$, the layer-polarization degree (LPD), for the electronic states in a BLP QD, which characterizes how much an electronic state is distributed over the top and bottom layers, and mathematically this quantity is given by

$$
\eta=\frac{\int\left|\Psi^{t}(r)\right|^{2} d r-\int\left|\Psi^{b}(r)\right|^{2} d r}{\int\left|\Psi^{t}(r)\right|^{2} d r+\int\left|\Psi^{b}(r)\right|^{2} d r}
$$

where $\Psi^{t}(r)$ and $\Psi^{b}(r)$ are the electronic probability densities in the top and bottom layers, respectively. Thus, $\eta=1(-1)$ indicates that the electronic states are only distributed over the top (bottom) layer (i.e., completely layer polarized), $\eta=0$ indicates that the electronic states are symmetrically distributed over the two layers (i.e., fully layer unpolarized), and $0<|\eta|<1$ indicates that the electronic states are asymmetrically distributed over the two layers (i.e., partially layer polarized).

In Fig. 4(a), we show the LPD-projected energy levels of the same BLP QD as in Fig. 3(a). The inset plot shows more clearly the edge-state levels that are very close to each other around zero energy. As can be seen, the edge states inside the band gap of the QD are partially layer polarized $(0<|\eta|<1)$ while the conduction and valence bulk states outside the band gap are fully layer unpolarized $(\eta=0)$. To clearly see such layer-polarized features of the bulk and edge states, we show in Figs. 4(b)-4(d) the layer-resolved electronic probability densities $\left|\Psi^{t}\right|^{2}$ and $\left|\Psi^{b}\right|^{2}$ of the bulk and 

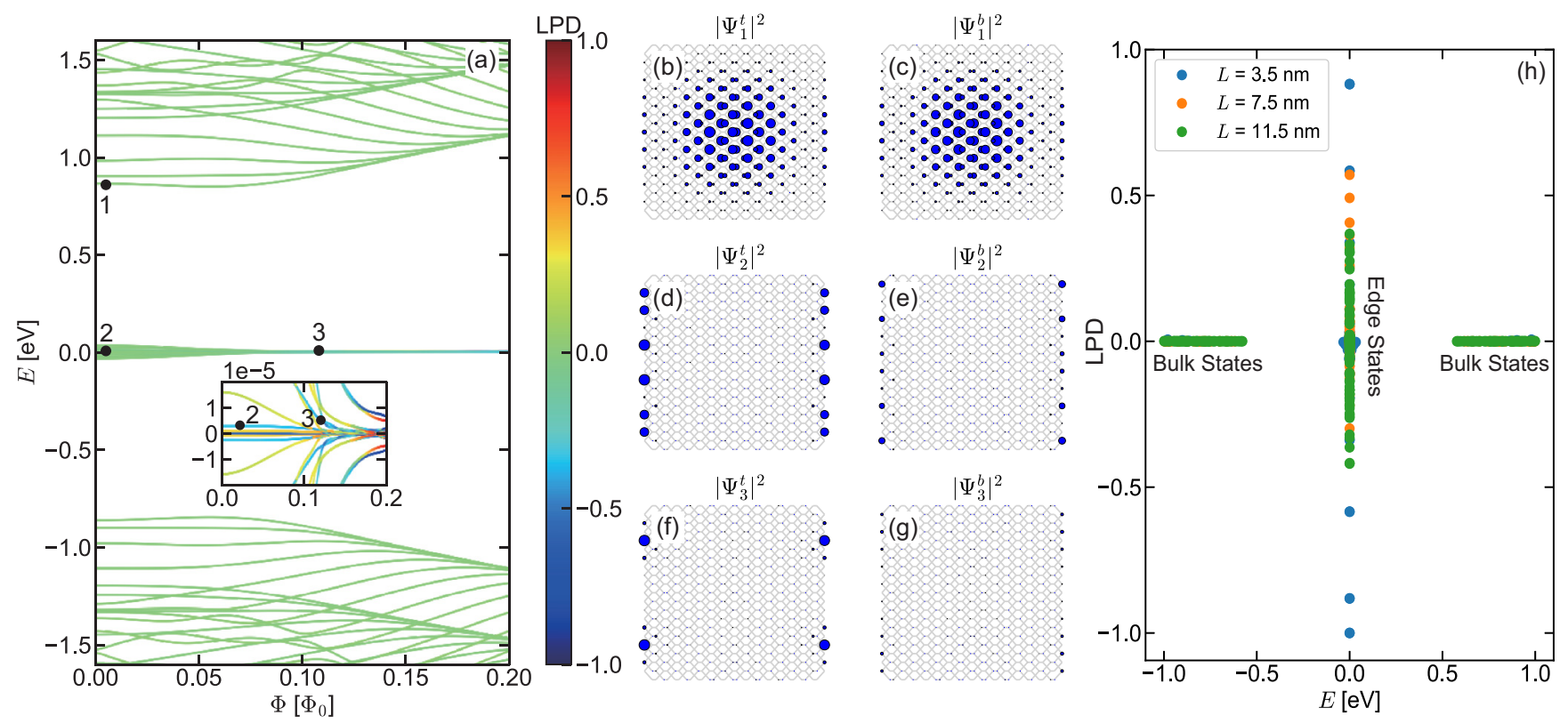

FIG. 4. (a) Energy levels of an unbiased BLP QD with dot size $L=3.5 \mathrm{~nm}$, as a function of the magnetic flux $\Phi$, where the colorbar shows the LPD $(\eta)$ values at these levels. The inset shown in (a) shows a zoom of the edge levels around zero energy. (b)-(g) Layer-resolved probability densities of the electronic states indicated by points $1-3$ shown in (a). $\left|\Psi_{j}^{t}\right|^{2}$ and $\left|\Psi_{j}^{b}\right|^{2}(j=1,2,3)$ denote the top-layer and bottom-layer electronic distributions, respectively. The size of the blue dots shown in (b)-(g) represents the amplitude of the electronic probability density. (h) Zero-field LPD $(\eta)$ values of the edge and bulk states for different QD sizes $L$ as indicated. There are many points around $E=0$ due to the highly quasidegenerate edge states around this zero energy.

edge states corresponding to points 1-3 marked in Fig. 4(a). Indeed, those edge states are partially layer polarized while those conduction and valence bulk states are fully layer unpolarized. Such a difference is mainly attributed to the different interlayer symmetries for the edge and bulk states, which can be understood as follows: When cutting the BLP sheet perpendicularly into squared-shaped QDs, we find that the presence of interlayer hoppings breaks the edge symmetry between the top and bottom phosphorene layers while it keeps the bulk symmetry between the two layers, as shown in Fig. 1(b). Notice that the edge atom of one layer is two hops away from the interlayer hopping $t_{3}$ while the other layer is only one hop away from it. Because edge states are strongly localized at the zigzag boundaries, the broken edge symmetry gives rise to zero-field layer polarization of the edge states. However, due to the bulk symmetry between the top and bottom phosphorene layers, the bulk states are symmetrically distributed over the two layers and thus there is no such layer polarization for the bulk states.

At lower magnetic fields, the edge states are less polarized due to their coupling to the unpolarized bulk states. With increasing magnetic field, they become more polarized because they are gradually decoupled from the bulk states (which become more localized around the QD center at high magnetic fields). Our numerical calculations also indicate a similar result for the larger-size QD, i.e., the edge (bulk) states are asymmetrically (symmetrically) distributed over the two layers and thus they are partially layer polarized (fully layer unpolarized). However, the difference is that the LPD values of the edge levels in the larger-size QD are almost unaffected by the magnetic field due to the absence of the bulk-edge coupling.
We also find that the zero-field LPD $(\eta)$ of edge states depends sensitively on the size of the BLP QD and its magnitude $(|\eta|)$ is increased by decreasing the QD size, as shown in Fig. 4(h). This is because the importance of dot boundaries is more significant in smaller-size QDs due to the increased edge-to-volume ratio. Note that there are many points around $E=0$ due to the highly quasidegenerate edge states around this zero energy. In addition, the zero-field LPD of edge states should also depend on the stacking order of BLP. For instance, (i) its sign is positive $\eta>0$ (or negative $\eta<0$ ) for the $\mathrm{AB}$ stacking, which may turn into negative $\eta<0$ (or positive $\eta>0$ ) for the BA stacking due to the inverted interlayer (boundary) symmetry (the two layers are just spatially flipped for the cases of $\mathrm{AB}$ and $\mathrm{BA}$ stackings); and (ii) its value for the $\mathrm{AB}$ (or $\mathrm{BA}$ ) stacking might be changed for the AA stacking due to the different interlayer (boundary) symmetries of these two stacking orders (the $\mathrm{AB}$ stacking has a relative in-plane shift between the two layers while the AA stacking does not).

\section{B. Bias effect}

Now we consider the effect of applying a perpendicular electric field (i.e., a bias potential) on the electronic properties of a BLP QD. Here, the bias potential energy $V$ is antisymmetric with respect to the $z=0$ plane, i.e., $V(z)=-V(-z)$, due to the mirror symmetry between the two layers in a BLP QD. In Figs. 5(a) and 5(b), we show the LPD-projected energy levels of a BLP QD at zero magnetic field $(\Phi=0)$, as a function of the bias potential $V$, for different dot sizes $L$ : (a) $L=3.5 \mathrm{~nm}$ and (b) $L=7.5 \mathrm{~nm}$. For comparative purposes, we also show in Fig. 5(c) the results of a MLP QD with dot size $L=7.5 \mathrm{~nm}$. 
(a)

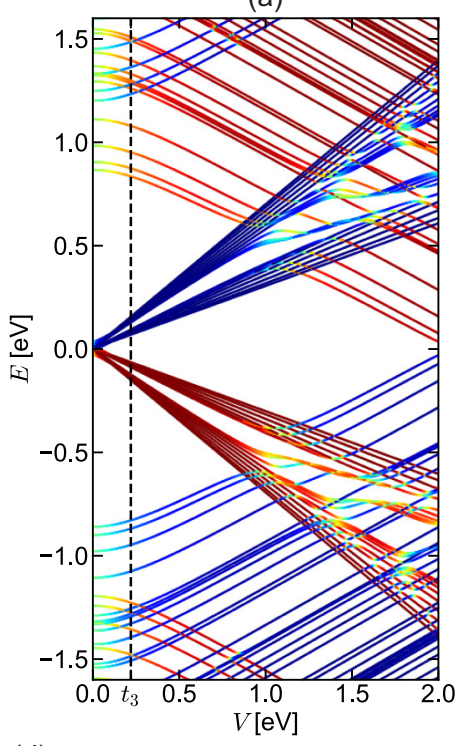

(b)

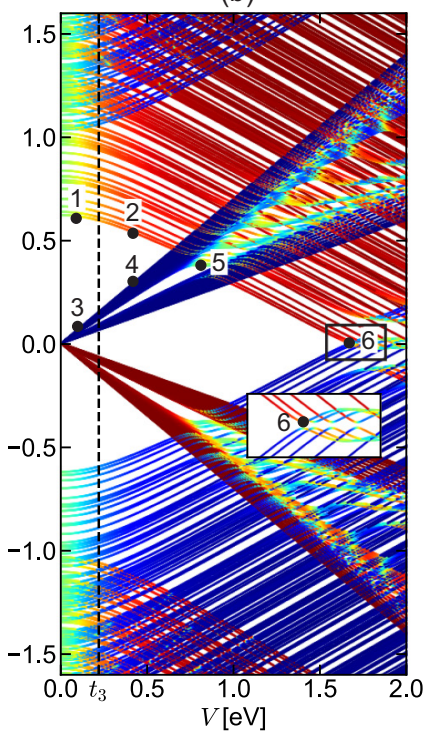

(c)

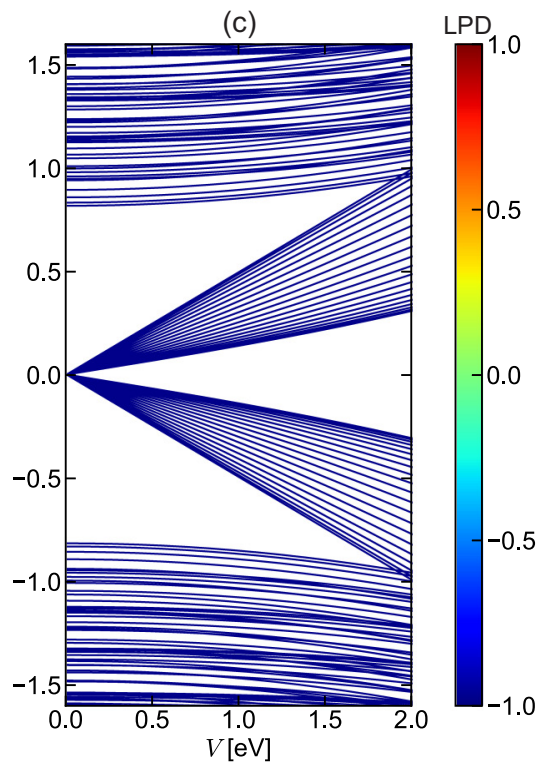

(d)

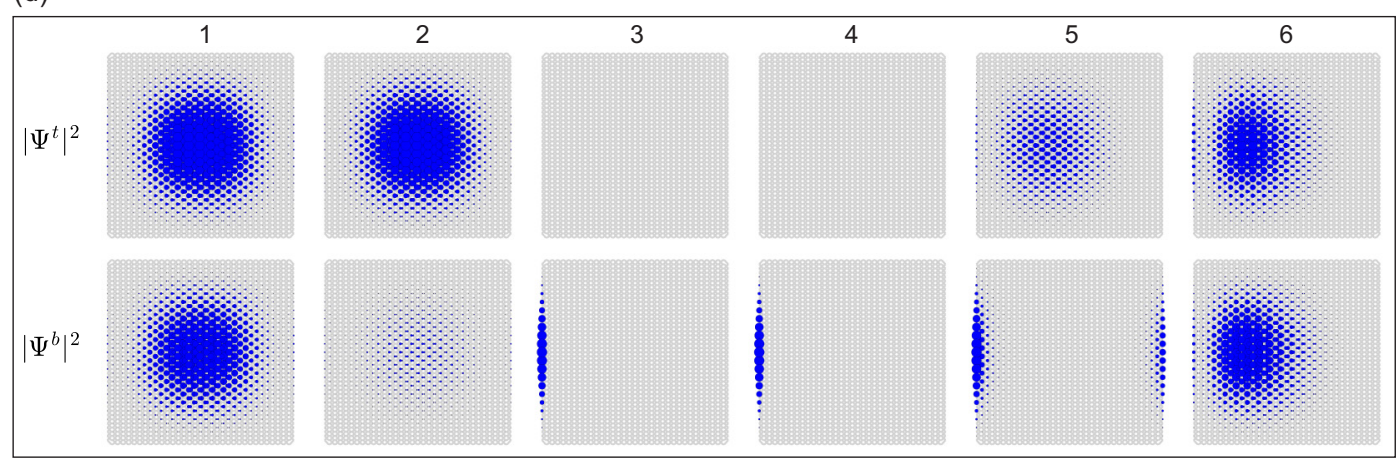

FIG. 5. (a) [(b)] LPD-projected energy levels of a BLP QD with dot size $L=3.5$ (7.5) nm, at zero magnetic field $(\Phi=0)$, as a function of the bias potential energy $V$. (c) Results of a MLP QD with dot size $L=7.5 \mathrm{~nm}$ shown for comparative purposes. (d) Layer-resolved probability densities $\left|\Psi^{t}\right|^{2}$ and $\left|\Psi^{b}\right|^{2}$ of the electronic states denoted by points 1-6 shown in (b). Here, the black rectangle shown in (b) is enlarged as an inset, and the vertical dashed lines shown in (a) and (b) mark the position at which the bias potential $V$ is equal to the interlayer coupling energy $t_{3}$.

As can be seen in Figs. 5(a) and 5(b), for smaller bias potentials the energy levels of the edge (bulk) states exhibit a linear (quadratic) Stark shift, while for larger bias potentials both the bulk and edge levels exhibit a linearlike Stark shift. We explain in the following the different behaviors of these Stark shifts observed for smaller and larger bias potentials.

The linear (quadratic) Stark effect exhibited by the edge (bulk) states observed for smaller bias potentials can be understood qualitatively within the framework of perturbation theory. As already shown in Figs. 3(a) and 3(b), the energy levels of the edge states are spaced very closely while those of the bulk states are not. Therefore, degenerate (nondegenerate) perturbation theory can be used to study the response of the edge (bulk) states to the external bias. For simplicity, we use a two-level model to explain the linear (quadratic) Stark effect observed for the edge (bulk) states in the BLP QD. Here for both the edge and bulk states, the two unperturbed (unbiased) energy levels are denoted by their eigenenergies $E_{1}$ and $E_{2}$ and corresponding eigenfunctions $|1\rangle$ and $|2\rangle$, and for the edge states $E_{1} \simeq E_{2}$ can be reasonably assumed due to their nearly degenerate energy levels. We further denote the edge-state or bulk-state Hamiltonian in the presence of an external bias as $H=H_{0}+V(z)$ with $H_{0}$ the unperturbed Hamiltonian and $V(z)$ the layer-dependent bias potential energy. For the unperturbed Hamiltonian $H_{0}$, we have $H_{0}|j\rangle=E_{j}|j\rangle(j=1$, 2 ) and we apply perturbation theory with respect to the bias potential $V(z)$.

Within nondegenerate perturbation theory, the energy of the perturbed bulk states can be obtained up to second order as

$$
E_{j}=E_{j}+\langle j|V(z)| j\rangle+\frac{|\langle j|V(z)| i\rangle|^{2}}{E_{j}-E_{i}},
$$

with $j=1,2$ and $i \neq j$. As mentioned previously, the unbiased bulk states have symmetric electronic distributions over the two layers, i.e., their wave functions are symmetric with respect to the $z=0$ plane. However, the perturbation (bias) potential energy is antisymmetric with respect to the $z=$ 0 plane, namely, $V(z)=-V(-z)$. Therefore, the first-order term in Eq. (3) is zero while the second-order one is nonzero. This simple result may qualitatively explain the quadratic Stark effect observed for the bulk states in the lower bias potential region. The perturbed edge-state Hamiltonian matrix $H$ can be obtained within first-order degenerate perturbation theory, in a 
basis set composed of unperturbed eigenfunctions $\{|1\rangle,|2\rangle\}$ as

$$
[H]=\left[\begin{array}{ll}
E_{0} & \Lambda \\
\Lambda^{\dagger} & E_{0}
\end{array}\right],
$$

where $E_{0}=E_{1}=E_{2}$ and $\Lambda=\langle 1|V(z)| 2\rangle$. Diagonalizing this Hamiltonian matrix, we obtain the energy levels of the edge states under the bias perturbation as $E_{ \pm}=E_{0} \pm|\Lambda|$, which may qualitatively explain the linear Stark effect observed for the edge states in the lower bias potential region.

However, for larger bias potentials, perturbation theory is no longer valid and can not be applied to explain the observed linearlike Stark shifts for both the bulk and edge states in the BLP QD. In this case, one has to resort to the TB Hamiltonian itself [Eq. (1)], where the bias potential energy is included as the on-site energy. In the presence of a bias potential, both the center and boundary atoms in the top (bottom) layer gain the same on-site energy $+V / 2(-V / 2)$. That is why both the bulk and edge states in the BLP QD exhibit a linearlike Stark effect at large bias potentials. Furthermore, because a large bias potential makes both states layer polarized, all the top-layer (bottom-layer) states feel only the on-site energy $+V / 2(-V / 2)$ and thus their energy levels move up (down) with increasing $V$ [see the blue and red curves shown in Figs. 5(a) and 5(b)]. However, this is not the case for the bulk and edge states in the MLP QD due to the absence of the layerpolarization feature. Because all the bulk/edge states are distributed only over one layer and they feel the same on-site potential $+V / 2$ (or $-V / 2$ ), their energy levels move up (or down) with increasing $V$, as shown in Fig. 5(c).

It is worth noting that in Figs. 5(a) and 5(b) the energy levels of the edge states in the biased BLP QD are split into four branches. However, the energy levels of the edge states in the biased MLP QD are only split into two such branches [see Fig. 5(c)]. This difference is attributed to the interlayer coupling effect. The four branches of edge states in the BLP QD have opposite linear Stark responses to the external bias: two of them go up while the other two go down, as the bias potential energy increases. Likewise, the conduction and valence bulk states in the BLP QD have opposite quadratic Stark responses to the external bias. For instance, the energy of the lowest (highest) conduction (valence) bulk states decreases (increases) with the bias potential energy, thereby leading to a decrease in the band gap of the BLP QD. Therefore, those two branches of edge states that move up (down) with increasing bias potential energy eventually merge into the conduction (valence) bulk states at large bias potentials. Notice that this merging may lead to anticrossings between the energy levels of those bulk and edge states [see Fig. 5(a)]. These anticrossings are more pronounced for the smaller BLP QD because of the larger bulk-edge coupling as mentioned above. The energy levels before or after anticrossings correspond to the pure bulk and edge states, while at anticrossings they correspond to the mixed bulk/edge states.

It is also of interest to look at the LPD $(\eta)$ values of the energy levels of the bulk and edge states shown in Fig. 5(b). From the line colors that characterize the $\eta$ values, we observe the following features: (i) The bulk states are weakly layer polarized when the bias potential energy is less than the interlayer coupling energy (i.e., $V<t_{3}$ ). (ii) With increasing bias potential energy such that $V>t_{3}$, the bulk states become strongly layer polarized. (iii) The edge states are always layer polarized whether $V<t_{3}$ or $V>t_{3}$. (iv) Once the edge states merge into the bulk states, their mixed states become less layer polarized as compared to the unmixed bulk and edge states. (v) At large bias potential energies, the lowest conduction bulk states are coupled to the highest valence bulk states, leading to anticrossings between their energy levels [see the inset shown in Fig. 5(b)]. Although both the uncoupled conduction and valence bulk states are layer polarized, the coupled conduction-valence bulk states are layer unpolarized due to the electron-hole symmetry of the energy spectrum.

To see the above features more clearly, we choose typical bulk and edge states marked by points 1-6 shown in Fig. 5(b) and plot their layer-resolved probability densities in Fig. 5(d). As can be seen, the bulk state corresponding to point 1 has almost equal electronic distribution over the top and bottom layers, implying that the bulk states are almost layer unpolarized when $V<t_{3}$, which is due to the dominant interlayer coupling effect. When $V>t_{3}$, the bulk states become layer polarized because of the stronger bias effect (see the result corresponding to point 2). However, the biased edge states are always layer polarized whether $V<t_{3}$ or $V>t_{3}$, which is almost unaffected by the bias potential energy (see the results corresponding to points 3 and 4). Once the edge states merge into the bulk states, their mixed states have finite electronic distributions in both the top and bottom layers (see the result corresponding to point 5), implying that the mixed states are less layer polarized as compared to the unmixed individual states. At large bias potentials, the layer-polarized conduction bulk states are coupled to the layer-polarized valence bulk states. The coupled conduction-valence bulk states have almost equal electronic distribution over the two layers (see the result corresponding to point 6), implying that they are layer unpolarized.

Additionally, when comparing Figs. 5(b) and 5(c), we find that the band gap of the BLP (MLP) QD decreases (increases) with increasing bias potential energy. This is a consequence of the different bias-potential-energy dependencies of their bulk counterparts (i.e., infinite BLP and MLP; see Fig. 2). However, unlike infinite BLP shown in Fig. 2, the band gap of the BLP QD cannot be closed completely with increasing bias potential energy [see the inset shown in Fig. 5(b)]. This difference is clearly induced by the finite-size effect in the BLP QD.

\section{Biased magnetic-field effect}

In Figs. 6(a)-6(c), we show the DOS-projected energy levels of a BLP QD with dot size $L=5.5 \mathrm{~nm}$, as a function of the magnetic flux $\Phi$, for different bias potential energies $V$ : (a) $V=0 \mathrm{eV}$, (b) $V=0.5 \mathrm{eV}$, and (c) $V=2 \mathrm{eV}$. As can be seen, in the presence of a finite bias potential, the single unbiased edge band is split into four individual bands and the corresponding biased edge states are not only layer resolved but also boundary resolved. To show this, we choose four typical biased edge states indicated by points 1-4 shown in Fig. 6(b) and plot their corresponding probability densities over the two layers in Fig. 6(d). Clearly, these four biased edge states are localized at the different zigzag boundaries and are distributed over the different layers. Their layer- and boundary-resolved 
(a)

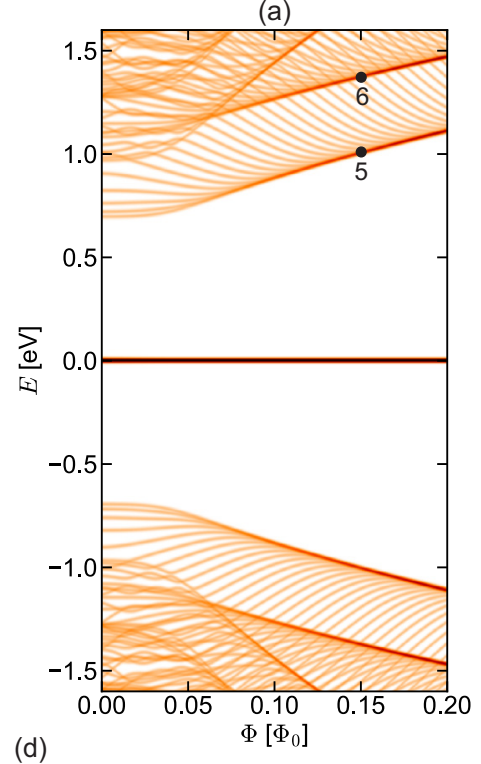

(b)

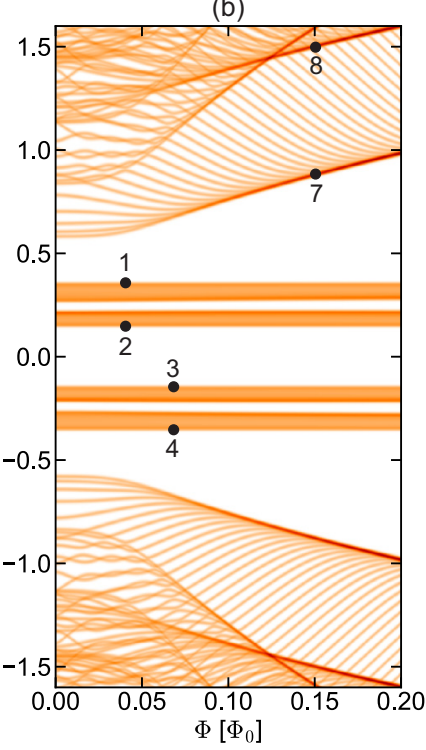

(c)



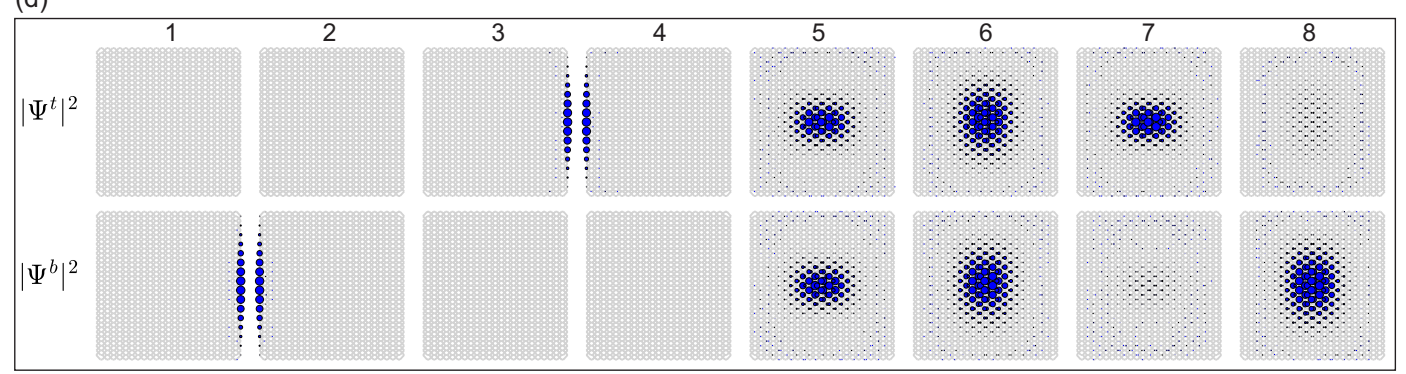

FIG. 6. DOS-projected energy levels of a BLP QD with dot size $L=5.5 \mathrm{~nm}$, as a function of the magnetic flux $\Phi$, for different bias potential energies $V$ : (a) $V=0 \mathrm{eV}$, (b) $V=0.5$, and (c) $V=2 \mathrm{eV}$. (d) Layer-resolved probability densities $\left|\Psi^{t}\right|^{2}$ and $\left|\Psi^{b}\right|^{2}$ of the electronic states denoted by points 1-8 shown in (a) and (b). The size of the blue dots shown in (d) represents the amplitude of the electronic probability density.

features are induced by the combined effects of perpendicular electric field and a puckered phosphorene lattice, introducing electrostatic on-site potential energies in a biased BLP QD that are not only layer dependent but also sublayer dependent.

With increasing bias potential energy from $V=0$ to $0.5 \mathrm{eV}$, the bulk LLs at high magnetic fields become layer polarized [see Fig. 6(d) corresponding to points 5-8 shown in Figs. 6(a) and 6(b)]. The biased bulk-LL states marked by points 7 and 8 shown in Fig. 6(b) have very different layer-resolved characteristics: one of them (the other) is only distributed over the top (bottom) layer. This feature indicates that there exist two groups of biased bulk LLs with distinctive electronic distributions over the two layers. When looking at the magnetic-field dependence of the bulk LLs formed at large magnetic fields, as shown in Figs. 6(a) and 6(b), we find that both unbiased and biased LLs exhibit a linearlike dependence on $\Phi$, which is characteristic of conventional $2 \mathrm{D}$ electronic systems with paraboliclike energy bands.

With further increasing bias potential energy to $V=2 \mathrm{eV}$, the bulk system of BLP (i.e., infinite BLP) becomes a Dirac-like semimetal, as shown in Fig. 2(c). This Diraclike semimetal phase of biased BLP features a gapless linearlike band dispersion along the $\Gamma-Y$ direction, a gapped paraboliclike energy spectrum along the $\Gamma-X$ direction, and an inverted band gap at the $\Gamma$ point due to the inversion of valence and conduction bands around this point. Similar interesting results were also demonstrated in Ref. [41], which studied electric-field tunable Dirac semimetal states in phosphorene multilayers with density functional theory. However, the confinement effect in the BLP QD opens a finite band gap at zero magnetic flux or field $(\Phi=0)$ [see Fig. 6(c)]. As the magnetic flux $\Phi$ increases, this band gap is first closed, leading to a semiconductor-to-semimetal transition, similar to that observed in Dirac material systems such as graphene QDs [42]; and then it is reopened, leading to a semimetal-to-semiconductor transition, similar to that observed in semiconductor material systems with paraboliclike band inversions such as InAs/GaSb broken-gap quantum wells [43]. Such two consecutive phase transitions induced by the magnetic field arise due to the coexistence of the gapless linearlike dispersion and the gapped paraboliclike spectrum in biased BLP, as shown in Fig. 2(c). At higher magnetic fields, a significantly large band gap can be observed. We note that inside this band gap no edge states are present because they merge into the bulk states outside the band gap. Moreover, we find in the presence of a large bias potential energy $(V=2 \mathrm{eV})$ and for larger magnetic fields that the zeroth bulk LLs exhibit a linearlike dependence on the magnetic field, while the others exhibit a square-root-like dependence [see Fig. 6(c)]. This feature is remarkably different than that shown in Figs. 6(a) and 
6(b), which is also attributed to the coexistence of the gapless Dirac-like dispersion and the gapped paraboliclike spectrum in biased BLP.

\section{CONCLUDING REMARKS}

By means of the TB approach, we have investigated the electronic properties of BLP QDs in the presence of perpendicular electric and magnetic fields. The energy levels, wave functions, and density of states of BLP QDs were obtained as a function of magnetic field and of bias potential. We find in-gap edge states that are well separated from gapped bulk states. The edge states are strongly localized at the zigzag boundaries of the QD and, as a result, they are almost unaffected by the magnetic field, while the bulk states are mainly distributed around the center part of the QD and thus they are strongly affected by the magnetic field, resulting in distinct LLs at high magnetic fields. However, both the edge and bulk states are found to be strongly influenced by the bias potential. For instance, their energy levels exhibit remarkably linear and quadratic Stark shifts, respectively. The different Stark effects exhibited by the edge and bulk states are qualitatively explained by using perturbation theory.

The size effect on the bulk and edge states in BLP QDs was also investigated. We found that in smaller-sized BLP QDs the edge states couple to the bulk states. Such a bulk-edge coupling decreases and eventually disappears with increasing magnetic field, because the bulk states become more confined due to the strong magnetic confinement while the edge states are almost unaffected by the magnetic field.

Since BLP is composed of two coupled phosphorene layers, the bulk and edge states in BLP QDs show interesting layer-dependent electronic properties, such as layer-resolved electronic distributions and their layer-polarization features, in the absence and presence of perpendicular electric and magnetic fields. We find that in the absence of a bias potential only edge states are layer polarized while the bulk states are not. The layer-polarization degree of edge states increases with increasing magnetic field and decreases with increasing QD size. However, in the presence of a bias potential both the edge and bulk states are layer polarized, and the layer-polarization degrees of the bulk (edge) states depend strongly (weakly) on the interplay of the bias potential and the interlayer coupling. The layer-polarization features of the edge and bulk states are clearly demonstrated by their layer-resolved electronic distributions. At high magnetic fields, the applied bias renders the bulk electrons in a BLP QD to perform cyclotron motion mainly in the bottom or top layer, leading to layer-polarized bulk LLs, and consequently there are two groups of biased bulk LLs with distinctive layer-resolved electronic distributions.

We also found that, in the presence of a large bias potential, semiconducting bulk BLP becomes a Dirac-like semimetal with a paraboliclike band inversion. As a consequence, with increasing magnetic field, the band gap of the BLP QD is first closed, leading to a semiconductor-to-semimetal transition, similar to that observed in Dirac material systems such as graphene QDs, and then it is reopened, leading to a semimetalto-semiconductor transition, similar to that observed in semiconductor material systems with paraboliclike band inversions such as InAs/GaSb broken-gap quantum wells. Moreover, due to the coexistence of the gapless Dirac-like band dispersion and the inverted paraboliclike energy spectrum in biased BLP, at large magnetic fields the zeroth bulk LLs in biased BLP QDs exhibit a linearlike dependence on magnetic field while the other LLs exhibit a square-root-like dependence.

\section{ACKNOWLEDGMENTS}

This work was financially supported by the Flemish Science Foundation (FWO-Vl), the National Natural Science Foundation of China (Grant No. 11574319), and the Chinese Academy of Sciences.
[1] L. Li, Y. Yu, G. J. Ye, Q. Ge, X. Ou, H. Wu, D. Feng, X. H. Chen, and Y. Zhang, Nat. Nanotech. 9, 372 (2014).

[2] H. Liu, A. T. Neal, Z. Zhu, Z. Luo, X. Xu, D. Tomanek, and P. D. Ye, ACS Nano 8, 4033 (2014).

[3] J. R. Brent, N. Savjani, E. A. Lewis, S. J. Haigh, D. J. Lewis, and P. O. Brien, Chem. Commun. 50, 13338 (2014).

[4] L. Cartz, S. R. Srinivasa, R. J. Riedner, J. D. Jorgensen, and T. G. Worlton, J. Chem. Phys. 71, 1718 (1979).

[5] A. Morita, Appl. Phys. A 39, 227 (1986).

[6] A. S. Rodin, A. Carvalho, and A. H. Castro Neto, Phys. Rev. Lett. 112, 176801 (2014).

[7] F. Xia, H. Wang, and Y. Jia, Nat. Commun. 5, 4458 (2014).

[8] J. Qiao, X. Kong, Z. X. Hu, F. Yang, and W. Ji, Nat. Commun. 5, 4475 (2014).

[9] X. Chen, Y. Wu, Z. Wu, Y. Han, S. Xu, L. Wang, W. Ye, T. Han, Y. He, Y. Cai, and N. Wang, Nat. Commun. 6, 7315 (2015).

[10] L. Li, F. Yang, G. J. Ye, Z. Zhang, Z. Zhu, W. Lou, X. Zhou, L. Li, K. Watanabe, T. Taniguchi, K. Chang, Y. Wang, X. Hui Chen, and Y. Zhang, Nat. Nanotech. 11, 593 (2016).
[11] Y. Li, S. Yang, and J. Li, J. Phys. Chem. C 118, 23970 (2014).

[12] D. Çakir, H. Sahin, and F. M. Peeters, Phys. Rev. B 90, 205421 (2014).

[13] J. M. Pereira, Jr. and M. I. Katsnelson, Phys. Rev. B 92, 075437 (2015).

[14] S. Yuan, E. van Veen, M. I. Katsnelson, and R. Roldan, Phys. Rev. B 93, 245433 (2016).

[15] J.-Y. Wu, S.-C. Chen, G. Gumbs, and M.-F. Lin, Phys. Rev. B 95, 115411 (2017).

[16] V. Tran and L. Yang, Phys. Rev. B 89, 245407 (2014).

[17] X. Zhang, H. Xie, Z. Liu, C. Tan, Z. Luo, H. Li, J. Lin, L. Sun, W. Chen, Z. Xu, L. Xie, W. Huang, and H. Zhang, Angew. Chem. Int. Ed. 54, 3653 (2015).

[18] Z. Sun, H. Xie, S. Tang, X. F. Yu, Z. Guo, J. Shao, H. Zhang, H. Huang, H. Wang, and P. K. Chu, Angew. Chem. Int. Ed. 127, 11688 (2015).

[19] R. Zhang, X. Y. Zhou, D. Zhang, W. K. Lou, F. Zhai, and K. Chang, 2D Materials 2, 045012 (2015).

[20] X. Niu, Y. Li, H. Shu, and J. Wang, J. Phys. Chem. Lett. 7, 370 (2016). 
[21] L. L. Li, D. Moldovan, W. Xu, and F. M. Peeters, Nanotechnology 28, 085702 (2017).

[22] L. L. Li, D. Moldovan, P. Vasilopoulos, and F. M. Peeters, Phys. Rev. B 95, 205426 (2017).

[23] Y. Xu, J. Dai, and X. C. Zeng, J. Phys. Chem. Lett. 6, 1996 (2015).

[24] V. Wang, Y. C. Liu, Y. Kawazoe, and W. T. Geng, J. Phys. Chem. Lett. 6, 4876 (2015).

[25] G. Zhang, S. Huang, A. Chaves, C. Song, V. O. Ozcelik, T. Low, and H. Yan, Nat. Commun. 8, 14071 (2015).

[26] Z. Z. Zhang, Kai Chang, and F. M. Peeters, Phys. Rev. B 77, 235411 (2008).

[27] X. Y. Zhou, R. Zhang, J. P. Sun, Y. L. Zou, D. Zhang, W. K. Lou, F. Cheng, G. H. Zhou, F. Zhai, and Kai Chang, Sci. Rep. 5, 12295 (2015)

[28] D. Çakir, C. Sevik, and F. M. Peeters, Phys. Rev. B 92, 165406 (2015).

[29] A. N. Rudenko and M. I. Katsnelson, Phys. Rev. B 89, 201408(R) (2014).

[30] A. N. Rudenko, S. Yuan, and M. I. Katsnelson, Phys. Rev. B 92, 085419 (2015).

[31] K. Dolui and Su Ying Quek, Sci. Rep. 5, 11699 (2015).
[32] M. Ezawa, New J. Phys. 16, 115004 (2014).

[33] J.-W. Jiang and H. S. Park, Phys. Rev. B 91, 235118 (2015).

[34] D. Moldovan and F. M. Peeters, PYBINDING: A PYTHON package for tight-binding calculations, http://dx.doi.org/10.5281/zenodo.56818.

[35] D. Zhang, W. Lou, M. Miao, S.-C. Zhang, and K. Chang, Phys. Rev. Lett. 111, 156402 (2013).

[36] Steven S. Andrews and Steven G. Boxer, J. Phys. Chem. A 104, 11853 (2000).

[37] C. Song and P. Wang, Rev. Sci. Instrum. 81, 054702 (2010).

[38] B. Ostahie and A. Aldea, Phys. Rev. B 93, 075408 (2016).

[39] W. Li, G. Zhang, and Y.-W. Zhang, J. Phys. Chem. C 118, 22368 (2014).

[40] K. Kim, Sinisa Coh, C. Kisielowski, M. F. Crommie, Steven G. Louie, and M. L. Cohen, Nat. Commun. 4, 2723 (2013).

[41] B. Ghosh, B. Singh, R. Prasad, and A. Agarwal, Phys. Rev. B 94, 205426 (2016).

[42] A. D. Güçlü, P. Potasz, and P. Hawrylak, Phys. Rev. B 88, 155429 (2013).

[43] A. Zakharova, S. T. Yen, and K. A. Chao, Phys. Rev. B 69, 115319 (2004). 